\title{
How is price explosivity triggered in the cryptocurrency markets?
}

\author{
Yuzhi Cai ${ }^{1}$ - Thanaset Chevapatrakul ${ }^{2}$. Danilo V. Mascia ${ }^{3}$
}

Accepted: 9 September 2021 / Published online: 15 October 2021

(c) The Author(s) 2021

\begin{abstract}
We shed light on how the price explosivity characterising Bitcoin and other major cryptocurrencies is triggered, by employing the Quantile Self-Exciting Threshold Autoregressive (QSETAR) model. Our results for Bitcoin, Ripple, and Stellar reveal that the explosive behaviour originates from the extreme upper tails of the return distributions following a price increase in the preceding day. We do not find evidence of explositivity in the price of Litecoin.
\end{abstract}

Keywords Explosiveness · Cryptocurrencies · Bayesian methods · Quantile SETAR model

JEL Classification C21 · C51 · C53 · C58 · G12 · G15

\section{Introduction}

Much has been written about Bitcoin-and other cryptocurrencies-since its inception in 2008. As the related markets keep developing and maturing, it is important to delve more deeply into the dynamics characterising their functioning (Katsiampa et al. 2019a). For instance, despite the fact that Bitcoin and other cryptocurrencies have become increasingly popular, their price behaviour-still extremely volatile-does not seem to have been entirely comprehended as of yet (King and Koutmos 2021).

Danilo V. Mascia

D.V.Mascia@leeds.ac.uk

Yuzhi Cai

y.cai@swansea.ac.uk

Thanaset Chevapatrakul

Thanaset.Chevapatrakul@nottingham.ac.uk

1 Department of Accounting and Finance, School of Management, Swansea University, Bay Campus, Fabian Way, Swansea SA1 8EN, UK

2 Nottingham University Business School, University of Nottingham, Jubilee Campus, Nottingham NG8 1BB, UK

3 International Banking Institute, Leeds University Business School, University of Leeds, Maurice Keyworth Building, Leeds LS2 9JT, UK 


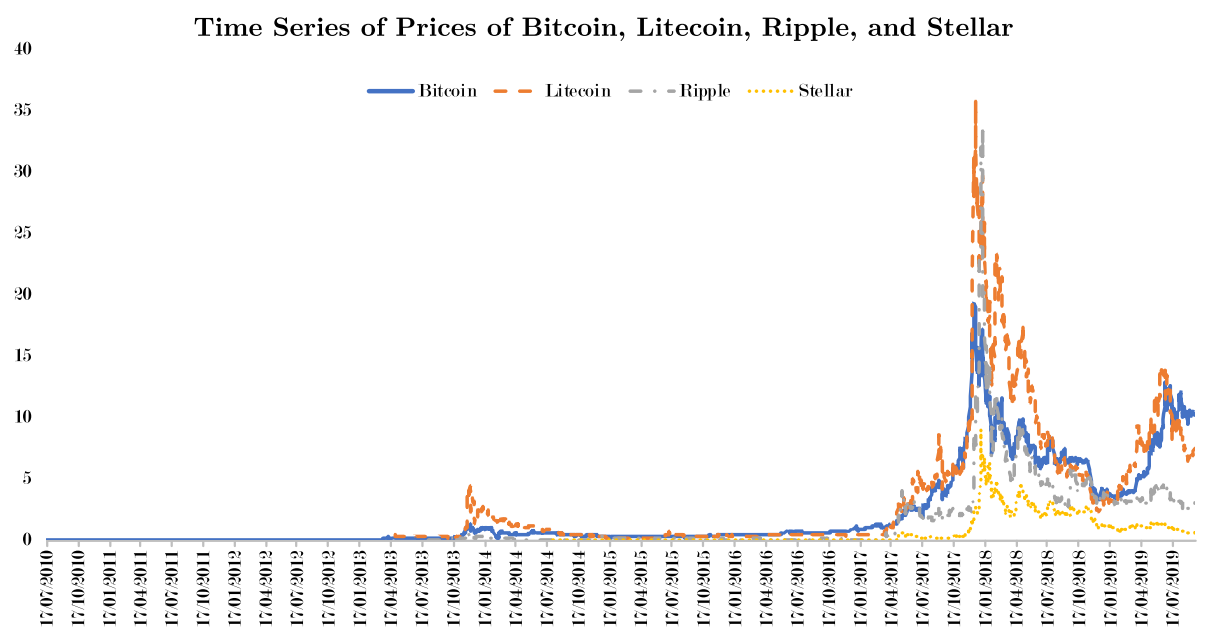

Fig. 1 Time series of prices of Bitcoin, Litecoin, Ripple, and Stellar. This figure plots the time series of prices of Bitcoin, Litecoin, Ripple, and Stellar. To ensure the readability and informativeness of the chart, we scale the prices, in a similar fashion to Borri (2019), as follows: we divide Bitcoin by 1,000, and Litecoin by 10; whereas we multiple both Ripple and Stellar by 10 . The sample for each of the sampled cryptocurrencies starts from the first available daily observation (i.e., 17/7/2010, 28/4/2013, 4/8/2013, and 5/8/2014, for Bitcoin, Litecoin, Ripple, and Stellar, respectively), until 19/09/2019

During the past three years, cryptocurrency enthusiasts witnessed a number of strong rallies in the Bitcoin markets. For example, the first rally started sometime in the beginning of October 2017 when the price of Bitcoin per US dollar (BTC/USD) rose sharply from around $\$ 4,000$ to about $\$ 20,000$ in mid-December-an increase of approximately $400 \%$ in a space of two and a half months. After the spectacular rise, BTC/USD steadily marched downward to reach its lowest point of just above \$3,200. Around the end of March 2019, the currency unexpectedly staged a comeback. The BTC/USD soared to about $\$ 12,000$ around August from approximately $\$ 4,000$ - an increase of roughly $200 \%$. During the periods of these two powerful market rallies, the Bitcoin market saw no fewer than 10 days of very large positive price jumps: daily returns of $20.34 \%$ realised on 7th December 2017 and $16.72 \%$ observed on 2nd April 2019, for example. ${ }^{1}$ The "bubble-like" and "explosive" behaviour of BTC/USD, clearly illustrated in Fig. 1, might be ascribed to the relative newness of the cryptocurrency markets and the consequent appeal that this generates on speculators (Bouri et al. 2019).

Originally designed to be an innovative and decentralised method of payment free from central banks' intervention, the excitement which Bitcoin sparked triggered off debates on whether it should be considered as a speculative vehicle (Baur et al. 2018) or an asset class to hold for hedging or diversification purposes (Panagiotidis et al. 2019). Besides that, scholars have also devoted particular attention to research on a number of other themes regarding Bitcoin and cryptocurrencies in general, such as volatility (Katsiampa 2017; Baur and Dimpfl 2018), volatility spillovers (Ji et al. 2019; Koutmos 2018; Corbet et al. 2018b), price jump risk (Chaim and Laurini 2018; Scaillet et al. 2018; Gronwald 2019), tail risk (Gkillas and

\footnotetext{
1 It is worth noting that our sample ends in September 2019, which is when we started working on this project. At the time of writing, we were aware of the most recent and still ongoing rallies in the Bitcoin markets. These rallies had started approximately in December 2020 and had pushed BTC/USD up to just over $\$ 40,000$ in January 2021, which is the all-time high. This period is not included in our analysis.
} 
Katsiampa 2018; Borri 2019), technical trading (Hudson and Urquhart 2021), herding and feedback trading (King and Koutmos 2021), price efficiency (Urquhart 2016; Nadarajah and Chu 2017; Bariviera 2017; Khuntia and Pattanayak 2018) and, more broadly, the importance of information in market activity (Katsiampa et al. 2019b). While much has been written on the inefficiency and on the exuberance of the cryptocurrency markets, more investigations are needed to study how observed "bubble-like" behaviours are brought about.

A number of scholars have looked into the explosivity theme characterizing the exuberance in cryptocurrency prices. More specifically, Corbet et al. (2018a) analyse the underline fundamentals (i.e., blockchain position, hashrate, and liquidity) that can significantly thrive price growth, and then employ such measures to detect and datestamp Bitcoin and Ethereum bubbles. The authors observe the existence of short periods in which each fundamental clearly impacts on the price formation, and nevertheless highlight that such effects vanish rapidly. Fry (2018) corroborates the existence of bubbles in the Bitcoin and Ethereum markets, and warns that booms and bust can occur even in the absence of a clear bubble. The presence of bubbles in the Bitcoin market is further supported by Cretarola and Figà-Talamanca (2021) who attribute their existence to the correlation between market attention (as proxied by the Google Search Volume Index) and Bitcoin returns. Cagli (2019) widens his sample and includes some more altcoins in order to detect potential price explosivity and pairwise comovements in their explosive behaviour. He finds all cryptocurrencies, except one, to show explosivity, as well as a number of co-explosive relationships in some altcoins pairs. Bouri et al. (2019) take a step further and observe that the explosivity in one cryptocurrency is likely due to the existence of explosive dynamics in other cryptocurrencies. They demonstrate that co-explosivity is not necessarily driven by the size of the cryptocurrencies. Finally, a recent paper by Gronwald (2019) reveals overwhelming evidence that cryptocurrency prices are characterised by (temporary) explosiveness and confirms that some cryptocurrency prices are explosive even if expressed in terms of Bitcoin and not, as conventionally, in USD. Overall, while in general scholars and experts confirm evidence of price explosivity (Corbet et al. 2018a; Fry 2018; Bouri et al. 2019; Cagli 2019), none of them however shed light on how the behaviour is brought about.

In this article, using the Quantile Self-Exciting Threshold Autoregressive (QSETAR) model, we characterise the bubble-like behaviour in the prices of Bitcoin and three other major cryptocurrencies: Litecoin, Ripple, ${ }^{2}$ and Stellar. ${ }^{3}$ The QSETAR model of Cai and Stander (2008) specifies the cryptocurrency return at the different quantiles of the return distribution to follow different autoregressive processes according to the pre-defined thresholds. Specifically, our framework examines how the cryptocurrency markets react to past price information when returns are located at the different quantiles with added flexibility that allows the reaction to vary depending on the state in which the markets were previously. The technique captures a more realistic trading behaviour: cryptocurrency traders enter the markets after the market direction has become clear. Evidence of price explosivity uncovered in this paper goes beyond the findings documented in Corbet et al. (2018a), Fry (2018), Cagli (2019).

Our empirical results for Bitcoin, Ripple, and Stellar show that the explosive behaviour originates only from the extreme right tail of the return distribution following a price increase in the preceding day. Taking Bitcoin as an example, we find that when the return is located at the 95th percentile after the return on Bitcoin turns positive on the preceding day, a rise of

\footnotetext{
${ }^{2}$ In this paper, for ease of readability, we use the term 'Ripple' (i.e., the name of a technology company) to refer to the cryptocurrency named 'XRP'. Indeed, 'XRP' is Ripple's cryptocurrency.

3 The term 'Stellar' is commonly used to identify Stellar's cryptocurrency, whose symbol is 'XLM'.
} 
$1 \%$ in the return is estimated to push the price up by a further $1.30 \%$. We do not find evidence of explosivity in the price of Litecoin, however.

In the following section, we present an exposition of the QSETAR model of Cai and Stander (2008). Sections 3 and 4 discuss our sample and the empirical results, respectively. Section 5 concludes.

\section{Methodology}

A general quantile self-exciting threshold autogressive (QSETAR) time series model (Cai and Stander 2008) is defined by

$$
q_{r_{t} \mid \boldsymbol{r}_{t-1}}^{\tau}=\sum_{i=1}^{m+1}\left(\beta_{i 0}^{\tau}+\beta_{i 1}^{\tau} r_{t-1}+\ldots+\beta_{i p}^{\tau} r_{t-p}\right) I_{\left[r_{t-d} \tau \in \Omega_{i}\right]}
$$

where $\Omega_{i}=\left(w_{i-1}, w_{i}\right], i=1, \ldots, m$, and $\Omega_{m+1}=\left(w_{m}, w_{m+1}\right)$, where $m \geq 0$ and $-\infty=w_{0}<w_{1}<\cdots<w_{m+1}=\infty$ are thresholds, $p$ is the order of the model, $\boldsymbol{r}_{t-1}=$ $\left(r_{t-1}, r_{t-2}, \ldots, r_{0}\right)^{T}, \beta_{i j}^{\tau}$ for $j=0,1, \ldots, p$ and $d^{\tau}$ are model parameters depending on $\tau$. Moreover, $\tau \in(0,1)$ is the probability such that $P\left(r_{t}<q_{r_{t} \mid \boldsymbol{r}_{t-1}}^{\tau} \mid \boldsymbol{r}_{t-1}\right)=\tau$ and $d^{\tau}$ is the delay parameter of the model. Therefore, $q_{r_{t} \mid r_{t-1}}$ is the $\tau$ th conditional quantile of the distribution of $r_{t}$.

Let $\beta^{\tau}=\left(\beta_{10}^{\tau}, \ldots, \beta_{1 p}^{\tau}, \ldots, \beta_{m+10}^{\tau}, \ldots, \beta_{m+1 p}^{\tau}, d^{\tau}\right)^{T}$. Then $\beta^{\tau}$ may be estimated by solving the following minimisation problem (Koenker 2005):

$$
\min _{\beta^{\tau}} \sum_{k+1}^{n} \rho_{\tau}\left(u_{t}\right)
$$

where $\rho_{\tau}\left(u_{t}\right)=u_{t}\left(\tau-I_{\left[u_{t}<0\right]}\right)$, and

$$
u_{t}=r_{t}-q_{r_{t} \mid r_{t-1}}^{\tau}=r_{t}-\sum_{i=1}^{m+1}\left(\beta_{i 0}^{\tau}+\beta_{i 1}^{\tau} r_{t-1}+\cdots+\beta_{i p}^{\tau} r_{t-p}\right) I_{\left[r_{t-d} \tau \in \Omega_{i}\right]},
$$

in which $k=\max \left\{p, d_{\max }\right\}$ and $d_{\max }$ is the maximum value of the delay parameter that one would like to consider. It is worth noting that Yu et al. (2010) suggest that for threshold GARCH models, $d_{\max }=3$ would be a reasonable choice. In this paper, we let $d_{\max }=5$ to cover a wider range of possible values of the delay parameter.

Since the delay parameter $d^{\tau}$ is an integer, it is not straightforward to solve the minimisation problem on the parameter space. However, it is worth noting that minimizing Eq. (2) is equivalent to maximising

$$
\ell\left(r_{k+1}, \ldots, r_{n} \mid \boldsymbol{\beta}^{\tau}, \mathbf{r}_{k}\right)=\tau^{n-k}(1-\tau)^{n-k} e^{-\sum_{t=k+1}^{n} \rho_{\tau}\left(u_{t}\right)} .
$$

This expression can be viewed as the likelihood function of $r_{k+1}, r_{k+2}, \ldots, r_{n}$ given $\mathbf{r}_{k}$ $=\left(r_{k}, r_{k-1}, \ldots, r_{0}\right)^{\top}$ if we assume that $r_{t}$ follows the threshold model

$$
r_{t}=\sum_{i=1}^{m+1}\left(\beta_{i 0}^{\tau}+\beta_{i 1}^{\tau} r_{t-1}+\cdots+\beta_{i p}^{\tau} r_{t-p}+\epsilon_{t}^{\tau}\right) I_{\left[r_{t-d} \tau \in \Omega_{i}\right]},
$$

where $\epsilon_{t}^{\tau}$ are iid asymmetric Laplace random variables with density function $f(\epsilon)=$ $\tau(1-\tau) e^{-\rho_{\tau}(\epsilon)}$. Therefore, the parameter estimates obtained by maximising the likelihood function, shown in Eq. (3), are the same as those obtained by minimising Eq. (2). 
Note that the involvement of the delay parameter $d^{\tau}$ will also make it difficult to solve the maximisation problem on the parameter space straightforward. We therefore adopt the Bayesian approach of Cai and Stander (2008) for the parameter estimation, whereby the posterior density function is given by

$$
\pi\left(\boldsymbol{\beta}^{\tau} \mid \mathbf{r}\right) \propto \ell\left(r_{k+1}, \ldots, r_{n} \mid \boldsymbol{\beta}^{\tau}, \mathbf{r}_{k}\right) \pi\left(\boldsymbol{\beta}^{\tau}\right)=\tau^{n-k}(1-\tau)^{n-k} e^{-\sum_{t=k+1}^{n} \rho_{\tau}\left(u_{t}\right)} \pi\left(\boldsymbol{\beta}^{\tau}\right)
$$

where $\pi\left(\boldsymbol{\beta}^{\tau}\right)$ is the prior density function.

The prior density function $\pi\left(\boldsymbol{\beta}^{\tau}\right)$ allows us to make use of possible prior information about the parameters when estimating parameters. Cai and Stander (2008) showed that the posterior density function is well defined on the parameter space for any prior density function. This is important because it guarantees that a Markov chain Monte Carlo (MCMC) method can be used for parameter estimation.

Following the work of Cai and Stander (2008), we employ a flat prior for the parameters and hence no prior information about the parameters is used for the parameter estimation. This is in fact one of the advantages of their method as in reality we usually do not have any information on the parameters. The basic idea of this method is as follows. First, given $\tau$ and the current parameter value $\boldsymbol{\beta}^{\tau}$, the next possible parameter value, denoted by $\boldsymbol{\beta}^{\prime}$, is proposed in which the delay parameter $d^{\prime}$ is simulated from a uniform distribution on $\left\{1, \ldots, d_{\text {max }}\right\}$, and any other element in $\boldsymbol{\beta}^{\prime}$ is simulated from a normal distribution, centered at its current value given in $\boldsymbol{\beta}^{\tau}$. Then, the proposed value $\boldsymbol{\beta}^{\prime}$ is accepted as the next parameter value with a probability given by $\min \left\{\frac{\pi\left(\boldsymbol{\beta}^{\prime} \mid \boldsymbol{r}\right)}{\pi\left(\boldsymbol{\beta}^{\tau} \mid \boldsymbol{r}\right)}, 1\right\}$. By repeating these steps multiple times, a sequence of model parameters can be generated. The Markov chain theory guarantees that the equilibrium distribution of the Markov chain is the posterior distribution defined by $\pi\left(\boldsymbol{\beta}^{\tau} \mid \boldsymbol{r}\right) .^{4}$

\section{Data}

This article focuses on four cryptocurrencies, currently actively traded in the markets: Bitcoin, Litecoin, Ripple, and Stellar. In selecting the cryptocurrencies to be included in our investigation, we adopt the following criteria. First, based on the data on coinmarketcap.com as of 19/9/2019, we focus on the top ten most capitalised crytpocurrencies which cover about $89 \%$ of the entire crytpocurrency markets. Second, to ensure a sufficiently long time series, we choose only the cryptocurrencies that have been actively traded during the last five years. After the filtering process, we end up with four cryptocurrencies: Bitcoin, Litecoin, Ripple, and Stellar.

Daily dollar returns on the cryptocurrencies are computed using price data collected either from coinmarketcap.com or www.CryptoCompare.com. Specifically, we rely on coinmarketcap.com to gather data for Litecoin, Ripple, and Stellar because this website provides cryptocurrency prices as the volume weighted average of hundreds cross currency pairs converted to USD (Wei 2018). The downside of it, however, is that prices are only available from April 2013, which is not ideal for Bitcoin provided that this pioneer cryptocurrency started being traded early in 2010. Therefore, limited to this case, we rely on www.CryptoCompare. com as the website offers daily information starting from 17/7/2010 - that is when Bitcoin was first traded. The sample for each of the cryptocurrencies thus starts from the first available daily observation and ends on 19/9/2019. More specifically, our samples for Bitcoin, Litecoin, Ripple, and Stellar start from 17/7/2010, 28/4/2013, 4/8/2013, and 5/8/2014, respectively.

$\overline{4}$ See Brooks (1998), for example. 
Table 1 Summary statistics of cryptocurrency returns

\begin{tabular}{|c|c|c|c|c|}
\hline & Bitcoin & Litecoin & Ripple & Stellar \\
\hline $\mathrm{N}$ & 3351 & 2335 & 2237 & 1871 \\
\hline Mean & 0.0037 & 0.0012 & 0.0018 & 0.0019 \\
\hline Median & 0.0019 & -0.0003 & -0.0027 & -0.0035 \\
\hline Std. deviation & 0.0665 & 0.0653 & 0.0740 & 0.0769 \\
\hline Skewness & 2.9480 & 1.7170 & 2.0510 & 1.9870 \\
\hline Std. error of skewness & 0.0420 & 0.0510 & 0.0520 & 0.0570 \\
\hline Kurtosis & 94.3830 & 25.2090 & 29.0410 & 16.0710 \\
\hline Std. error of Kurtosis & 0.0850 & 0.1010 & 0.1030 & 0.1130 \\
\hline Minimum & -0.8488 & -0.5139 & -0.6163 & -0.3664 \\
\hline Maximum & 1.4744 & 0.8290 & 1.0274 & 0.7231 \\
\hline
\end{tabular}

This table reports summary statistics for Bitcoin, Litecoin, Ripple, and Stellar. Our samples for Bitcoin, Litecoin, Ripple, and Stellar start from 17/7/2010, 28/4/2013, 4/8/2013, and 5/8/2014, respectively. The time series for all the cryptocurrencies in the sample ends on 19/9/2019

Table 2 Quantiles of cryptocurrency returns

\begin{tabular}{lrrrr}
\hline$\tau$ & Bitcoin & Litecoin & Ripple & \multicolumn{1}{c}{ Stellar } \\
\hline 0.05 & -0.0770 & -0.0839 & -0.0923 & -0.1024 \\
0.25 & -0.0136 & -0.0206 & -0.0230 & -0.0310 \\
0.50 & 0.0019 & -0.0003 & -0.0027 & -0.0035 \\
0.75 & 0.0218 & 0.0194 & 0.0203 & 0.0267 \\
0.95 & 0.0871 & 0.0912 & 0.1043 & 0.1166
\end{tabular}

This table reports the distributions of returns on Bitcoin, Litecoin, Ripple, and Stellar. We report the return values at the 5 th, 25th, 50th, 75th, and 95th quantiles. Our samples for Bitcoin, Litecoin, Ripple, and Stellar start from 17/7/2010, 28/4/2013, 4/8/2013, and 5/8/2014, respectively. The time series for all the cryptocurrencies in the sample ends on $19 / 9 / 2019$

Table 1 shows the number of observations available for each of the sampled cryptocurrencies along with some commonly used summary statistics. According to the reported statistics, Bitcoin has the highest daily mean return of around $0.4 \%$ and is the only cryptocurrency in the sample with positive median. The skewness and the kurtosis measures for all the cryptocurrencies in the sample point to the return distributions that are right-skewed and fattailed. Bitcoin, once again, has the highest values of both skewness and kurtosis as well as the largest maximum and the smallest minimum values. Complementing the descriptive statistics in Table 1, Table 2 reports the empirical distributions of returns, for each cryptocurrency, at different quantiles.

\section{Empirical analysis and results}

We estimate Eq. (1), setting $m=1, p=1, \ldots, 7, d_{\max }=5, \tau=0.05,0.25,0.50,0.75,0.95$. We fix $m=1$ because we are only interested in the conditional quantile function of the cryptocurrency return process when, subject to the delay parameter, the past return is located either above or below a pre-defined threshold. For each cryptocurrency, we employ two threshold 
Table 3 AIC statistics when the threshold is $w_{1}=0$

\begin{tabular}{|c|c|c|c|c|c|}
\hline \multicolumn{6}{|c|}{ Bitcoin } \\
\hline$p \backslash \tau$ & 0.05 & 0.25 & 0.50 & 0.75 & 0.95 \\
\hline 1 & 6.502108 & 7.629882 & 7.567755 & 7.982147 & 7.212924 \\
\hline 2 & 8.516421 & 9.367684 & 9.568465 & 9.864459 & 9.198823 \\
\hline 3 & 10.739352 & 11.322411 & 11.569194 & 11.858959 & 11.324587 \\
\hline 4 & 12.599169 & 13.288983 & 13.588904 & 13.699591 & 13.119390 \\
\hline 5 & 14.579397 & 15.343937 & 15.574193 & 15.739112 & 15.371966 \\
\hline 6 & 16.498389 & 17.269221 & 17.575322 & 17.725333 & 17.965440 \\
\hline 7 & 18.643327 & 19.343978 & 19.667224 & 19.781388 & 19.299285 \\
\hline \multicolumn{6}{|c|}{ Litecoin } \\
\hline$p \backslash \tau$ & 0.05 & 0.25 & 0.50 & 0.75 & 0.95 \\
\hline 1 & 6.309759 & 7.177583 & 7.400427 & 7.570019 & 7.029870 \\
\hline 2 & 8.554905 & 9.103804 & 9.420087 & 9.561237 & 9.343828 \\
\hline 3 & 10.343398 & 11.113302 & 11.380647 & 11.572815 & 11.497163 \\
\hline 4 & 12.357591 & 13.088285 & 13.391061 & 13.562340 & 13.110089 \\
\hline 5 & 14.312543 & 15.135724 & 15.383583 & 15.479943 & 15.243180 \\
\hline 6 & 16.395870 & 17.121087 & 17.395170 & 17.521574 & 17.428193 \\
\hline 7 & 18.336617 & 19.099428 & 19.396694 & 19.622497 & 18.999818 \\
\hline \multicolumn{6}{|c|}{ Ripple } \\
\hline$p \backslash \tau$ & 0.05 & 0.25 & 0.50 & 0.75 & 0.95 \\
\hline 1 & 6.265001 & 7.203146 & 7.404664 & 7.265546 & 6.544424 \\
\hline 2 & 8.464208 & 9.069515 & 9.474161 & 9.556994 & 8.581126 \\
\hline 3 & 10.204962 & 11.175128 & 11.490708 & 11.265592 & 10.597363 \\
\hline 4 & 12.345551 & 13.077693 & 13.416451 & 13.805275 & 12.715281 \\
\hline 5 & 14.159573 & 15.114419 & 15.572170 & 15.636419 & 15.634413 \\
\hline 6 & 16.255371 & 17.217220 & 17.392986 & 17.523048 & 17.328143 \\
\hline 7 & 18.858845 & 19.083811 & 19.387955 & 19.563025 & 19.472317 \\
\hline \multicolumn{6}{|c|}{ Stellar } \\
\hline$p \backslash \tau$ & 0.05 & 0.25 & 0.50 & 0.75 & 0.95 \\
\hline 1 & 6.183000 & 7.056618 & 7.401042 & 7.527477 & 6.734905 \\
\hline 2 & 8.616877 & 9.045981 & 9.405205 & 9.512064 & 8.667104 \\
\hline 3 & 10.722696 & 11.076436 & 11.437971 & 11.750134 & 11.187539 \\
\hline 4 & 12.044138 & 13.079619 & 13.430794 & 13.514415 & 13.465772 \\
\hline 5 & 14.067955 & 15.092446 & 15.612555 & 16.075160 & 15.087909 \\
\hline 6 & 16.089324 & 17.158386 & 17.434216 & 17.486799 & 17.358809 \\
\hline 7 & 18.406601 & 19.038180 & 19.400287 & 19.580555 & 19.544746 \\
\hline
\end{tabular}

This table shows the AIC statistics—-for each quantile $\tau$ and $p=1, \ldots, 7$-when the threshold value is $w_{1}=0$ 
Table 4 AIC statistics when the threshold is the median return

\begin{tabular}{|c|c|c|c|c|c|}
\hline \multicolumn{6}{|c|}{ Bitcoin } \\
\hline$p \backslash \tau$ & 0.05 & 0.25 & 0.50 & 0.75 & 0.95 \\
\hline 1 & 6.558470 & 7.401306 & 7.616606 & 7.811130 & 7.237105 \\
\hline 2 & 8.712559 & 9.377810 & 9.610050 & 9.769683 & 9.469767 \\
\hline 3 & 10.616625 & 11.374364 & 11.609519 & 11.836909 & 11.562212 \\
\hline 4 & 12.759651 & 13.365491 & 13.637782 & 13.894548 & 13.662482 \\
\hline 5 & 14.537339 & 15.315875 & 15.617206 & 15.774277 & 15.434152 \\
\hline 6 & 16.534188 & 17.319596 & 17.637874 & 17.830242 & 17.402648 \\
\hline 7 & 18.694055 & 19.332562 & 19.608678 & 19.787000 & 19.311141 \\
\hline \multicolumn{6}{|c|}{ Litecoin } \\
\hline$p \backslash \tau$ & 0.05 & 0.25 & 0.50 & 0.75 & 0.95 \\
\hline 1 & 6.287696 & 7.068629 & 7.438560 & 7.557180 & 6.984493 \\
\hline 2 & 8.493792 & 9.070666 & 9.356818 & 9.613444 & 9.924253 \\
\hline 3 & 10.146881 & 11.050838 & 11.356131 & 11.752736 & 11.710226 \\
\hline 4 & 12.154898 & 13.143162 & 13.355349 & 13.527987 & 13.620087 \\
\hline 5 & 14.151685 & 15.123064 & 15.401824 & 15.480751 & 15.616061 \\
\hline 6 & 16.095184 & 17.138353 & 17.359025 & 17.520704 & 17.356802 \\
\hline 7 & 18.546042 & 19.076425 & 19.369570 & 19.526664 & 19.432512 \\
\hline \multicolumn{6}{|c|}{ Ripple } \\
\hline$p \backslash \tau$ & 0.05 & 0.25 & 0.50 & 0.75 & 0.95 \\
\hline 1 & 6.112647 & 7.021669 & 7.350633 & 7.645379 & 6.497065 \\
\hline 2 & 8.174058 & 9.047064 & 9.397760 & 9.506547 & 9.251517 \\
\hline 3 & 10.278312 & 11.019051 & 11.348291 & 11.513425 & 11.406681 \\
\hline 4 & 12.830842 & 13.078399 & 13.350471 & 13.505912 & 13.777331 \\
\hline 5 & 14.187123 & 15.006143 & 15.373361 & 15.517410 & 15.169858 \\
\hline 6 & 16.114265 & 17.008317 & 17.344003 & 17.534641 & 17.712271 \\
\hline 7 & 18.230105 & 19.025180 & 19.526577 & 19.517259 & 19.315860 \\
\hline \multicolumn{6}{|c|}{ Stellar } \\
\hline$p \backslash \tau$ & 0.05 & 0.25 & 0.50 & 0.75 & 0.95 \\
\hline 1 & 6.079600 & 7.056956 & 7.356917 & 7.557868 & 6.925788 \\
\hline 2 & 8.107468 & 9.048540 & 9.409459 & 9.485658 & 8.623965 \\
\hline 3 & 9.911982 & 10.981165 & 11.357618 & 11.588873 & 11.567617 \\
\hline 4 & 11.984077 & 13.016580 & 13.357567 & 13.706924 & 13.672293 \\
\hline 5 & 14.085852 & 15.004719 & 15.436294 & 15.524615 & 15.305201 \\
\hline 6 & 16.028472 & 16.988928 & 17.352968 & 17.619626 & 17.016013 \\
\hline 7 & 18.137357 & 18.978120 & 19.447552 & 19.498815 & 20.011219 \\
\hline
\end{tabular}

This table shows the AIC statistics - for each quantile $\tau$ and $p=1, \ldots, 7$ - when the threshold value $w_{1}$ is the median return 
values in our analysis: $w_{1}=0$ and the cryptocurrency's median return. The threshold of zero allows the empirical analysis to mimic the real-world trading behaviour where cryptocurrency traders pay attention to whether or not the markets were positive or negative in the previous day. ${ }^{5}$ In addition, we also experiment with the cryptocurrency's median return as a threshold because it is conventional for researchers employing the quantile regression technique to estimate the response of the dependent variable to the covariates when the dependent variable is located at the median.

For each value of $p$, and each quantile level $\tau$, where $\tau=0.05,0.25,0.50,0.75,0.95$, we run-for each cryptocurrency - the MCMC estimation method from which we obtain, in total, 140 estimated models. For each estimated model, we collect 250 posterior samples for each parameter after a burn-in period. Then, for the delay parameter, we estimate its value by using the mode of its posterior samples and, for other parameters, we estimate their values by using the mean of the respective posterior samples. We also calculate the corresponding 95\% credible interval using the $2.5 \%$ and $97.5 \%$ quantiles of these posterior samples. The credible interval is an interval within which the model parameter falls with a 0.95 probability. Hence, if the interval contains number 0 , then we can say that the parameter is not significant. Moreover, for each estimated model, we also obtain the AIC statistics, according to which we find that the best fitted models-for both thresholds and for all the cryptocurrencies investigated-are the ones with $p=1$ (see, in this regard, Tables 3 and 4). This preliminary check, therefore, leads us to formalize the following model specification:

$$
q_{r_{t} \mid r_{t-1}}^{\tau}=\sum_{i=1}^{2}\left(\beta_{i 0}^{\tau}+\beta_{i 1}^{\tau} r_{t-1}\right) I_{\left[r_{t-d} \tau \in \Omega_{i}\right]} .
$$

We examine the model fit by employing the following procedures. Using the fitted models, we obtained the estimated quantiles at levels $\tau=0.05,0.25,0.5,0.75,0.95$. In Fig. 2, we present the observed return of the cryptocurrency, along with the estimated quantiles at the $5 \%$ and the $95 \%$ levels in black, green, and yellow, respectively. It can be seen that these quantile curves reflect the evolution of each cryptocurrency in our sample. In particular, Fig. 2 demonstrates that $5 \%$ of the return realisation for each cryptocurrency in the sample, as represented by the black line, are expected to be higher than the yellow line and 5\% of them are expected to be lower than the green line.

To test this, we calculate the percentages of the observed cryptocurrency that are under each of the five estimated quantile curves. We then calculate the mean squared errors (MSE) to measure the differences between these percentages and the actual $\tau$ value. We find that the MSE values are given by $0.00036,0.00094,0.00148$ and 0.00265 for Bitcoin, Litecoin, Ripple and Stellar, respectively_-suggesting that these models explain the data well.

We report our estimation results in Tables 5, 6, and 7. For each $\tau$ value, the means of the posterior samples of $\beta_{11}$ and $\beta_{21}$ are reported in the top row, and the associated $95 \%$ credible intervals are reported in the corresponding second and third rows. According to the results reported in Tables 5 and 6, we find no evidence of the explosive price behaviour in the Litecoin market while the evidence exists in the markets of the other cryptocurrencies, depending on the value of $w_{1}$. When $w_{1}=0$, the means of the posterior distributions of $\beta_{11}$ and $\beta_{21}$ for Bitcoin and Ripple, seen in Table 5, indicate that the explosive price behaviour is triggered when the rally, following the previous day's price increase, is strong enough for the returns to reach the 95th quantile. As an example, the value of $\beta_{21}$ of 1.2916 for Bitcoin at

\footnotetext{
5 This approach has earlier been adopted by Cai and Stander (2008) who investigate the time series property of the US GNP using the QSETAR model, also setting $m=1$. In their application, the US economy is assumed to behave in a different manner after contraction and expansion.
} 
Estimated Quantile Curves at the 5\% and the 95\% Quantiles
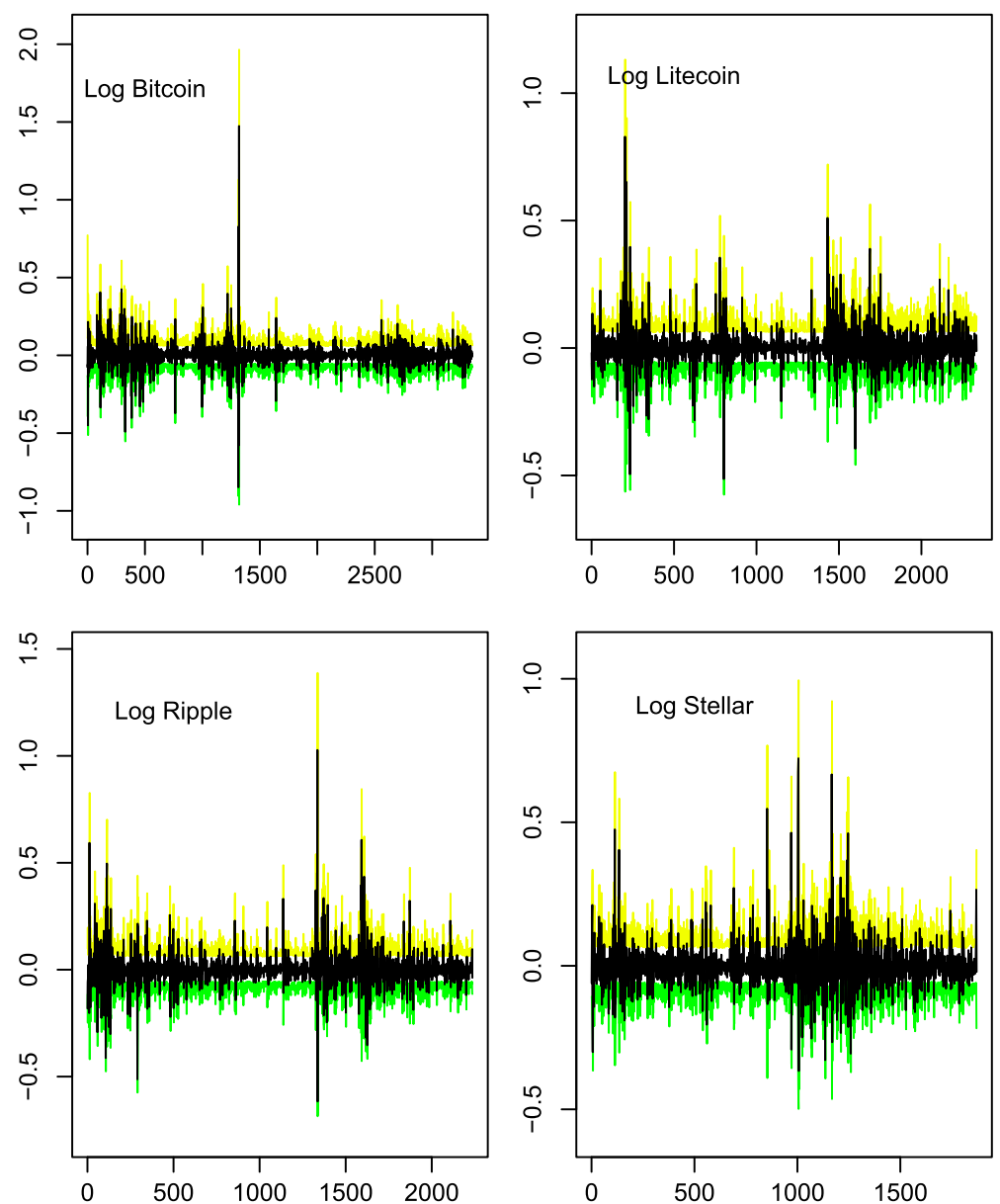

Fig. 2 Estimated quantile curves at the 5\% and the 95\% Quantiles. Estimated quantile curves at the 5\% (green) and $95 \%$ (yellow) levels for each cryptocurrency, where the black curves are the observed cryptocurrency $-5 \%$ of which are expected to be above the yellow line, and $5 \%$ of which are expected to be below the green line

$\tau=0.95$, reported in Table 5, indicates that one day after the return on Bitcoin turns positive, an increase of $1 \%$ in the return-when the return is located at the 95 th percentile-results in a further increase in return of around $1.30 \% .{ }^{6}$ When the threshold is the median return, the findings reported in Table 6 suggest that only Ripple and Stellar exhibit the explosive price behaviour at the 95th quantile one day after the previous day's return crosses the median return from below. We find no evidence of explosivity in the Bitcoin nor the Litecoin markets when the value of $w_{1}$ is set to be the cryptocurrency's median return.

Our empirical analysis complements and confirms previous research on price explosivity and co-explosivity by Bouri et al. (2019), Cagli (2019), and Gronwald (2021). While

6 The estimated value of $\beta_{21}$ is statistically significant because the credible interval for $\beta_{21}$ does not contain the value of 0 . In other words, the probability for $\beta_{21}$ taking positive values is $95 \%$. 
Table 5 The QSETAR estimation results

\begin{tabular}{|c|c|c|c|c|c|c|c|c|}
\hline \multirow[t]{2}{*}{$\tau$} & \multicolumn{2}{|l|}{ Bitcoin } & \multicolumn{2}{|l|}{ Litecoin } & \multicolumn{2}{|l|}{ Ripple } & \multicolumn{2}{|l|}{ Stellar } \\
\hline & $\beta_{11}$ & $\beta_{21}$ & $\beta_{11}$ & $\beta_{21}$ & $\beta_{11}$ & $\beta_{21}$ & $\beta_{11}$ & $\beta_{21}$ \\
\hline \multirow[t]{3}{*}{0.05} & 0.9855 & -0.6140 & 0.4085 & -0.4334 & 0.4761 & -0.7138 & 0.2939 & -0.5502 \\
\hline & -0.3503 & -1.8599 & -0.8130 & -1.6651 & -0.4759 & -2.4586 & -0.8247 & -2.8612 \\
\hline & 2.7027 & 0.3126 & 2.2672 & 0.4692 & 2.5605 & 0.3373 & 2.2639 & 0.4234 \\
\hline \multirow[t]{3}{*}{0.25} & 0.2623 & -0.1867 & 0.0700 & -0.1081 & 0.0869 & -0.1634 & -0.0943 & -0.1713 \\
\hline & -0.3371 & -0.7970 & -0.5166 & -0.5637 & -0.4961 & -0.6801 & -0.6292 & -0.7942 \\
\hline & 1.0691 & 0.2819 & 0.7971 & 0.3213 & 1.0846 & 0.3200 & 0.4969 & 0.3547 \\
\hline \multirow[t]{3}{*}{0.50} & 0.0064 & 0.0141 & -0.0611 & -0.0710 & -0.0395 & 0.0087 & -0.1284 & -0.0594 \\
\hline & -0.3996 & -0.3174 & -0.4470 & -0.5079 & -0.4890 & -0.3332 & -0.5955 & -0.5000 \\
\hline & 0.4250 & 0.4661 & 0.3938 & 0.3846 & 0.3624 & 0.4690 & 0.3423 & 0.4446 \\
\hline \multirow[t]{3}{*}{0.75} & -0.2447 & 0.3145 & -0.2442 & 0.4009 & -0.3373 & 0.6761 & -0.1883 & 0.3748 \\
\hline & -0.9714 & -0.2530 & -0.8415 & -0.3302 & -0.9310 & -0.1877 & -0.7581 & -0.3782 \\
\hline & 0.2869 & 1.0992 & 0.4006 & 1.3946 & 0.2096 & 1.7699 & 0.3187 & 1.4293 \\
\hline \multirow[t]{3}{*}{0.95} & -0.7315 & 1.2916 & -0.6520 & 1.0852 & -0.8664 & 1.7142 & -0.5724 & 1.6340 \\
\hline & -2.2899 & 0.0161 & -2.2344 & -0.3370 & -2.5596 & 0.1047 & -2.5536 & -0.0525 \\
\hline & 0.1806 & 3.4924 & 0.6113 & 2.8898 & 0.4952 & 3.5153 & 0.8000 & 3.7254 \\
\hline
\end{tabular}

This table reports the estimated parameter values for the QSETAR model shown in Eq. (4) where $p=1$, $d=1$, and $w_{1}=0$. For each value of $\tau$, where $\tau=0.05,0.25,0.50,0.75,0.95$, we report the means of the posterior distributions of parameters $\beta_{11}$ and $\beta_{21}$ (first row) along with the credible intervals which are shown below the corresponding mean estimates (second and third rows). Bold font is used to highlight statistical significance

Table 6 The QSETAR estimation results

\begin{tabular}{|c|c|c|c|c|c|c|c|c|}
\hline \multirow[t]{2}{*}{$\tau$} & \multicolumn{2}{|l|}{ Bitcoin } & \multicolumn{2}{|l|}{ Litecoin } & \multicolumn{2}{|l|}{ Ripple } & \multicolumn{2}{|l|}{ Stellar } \\
\hline & $\beta_{11}$ & $\beta_{21}$ & $\beta_{11}$ & $\beta_{21}$ & $\beta_{11}$ & $\beta_{21}$ & $\beta_{11}$ & $\beta_{21}$ \\
\hline \multirow[t]{3}{*}{0.05} & 0.8701 & -0.5122 & 0.3414 & -0.5104 & 0.4652 & -0.5437 & 0.2950 & -0.4006 \\
\hline & -0.2267 & -1.5665 & -0.9416 & -1.9766 & -0.7190 & -1.6411 & -0.8194 & -1.5886 \\
\hline & 2.3418 & 0.3224 & 1.9459 & 0.5003 & 2.0469 & 0.2439 & 2.8161 & 0.2792 \\
\hline \multirow[t]{3}{*}{0.25} & 0.1585 & -0.1348 & 0.0752 & -0.1505 & 0.0370 & -0.1550 & -0.0831 & -0.1703 \\
\hline & -0.4028 & -0.5866 & -0.4801 & -0.7362 & -0.5007 & -0.6360 & -0.6592 & -0.7836 \\
\hline & 0.9300 & 0.2508 & 0.9163 & 0.2512 & 0.7249 & 0.3089 & 0.4746 & 0.3413 \\
\hline \multirow[t]{3}{*}{0.50} & 0.0030 & -0.0180 & -0.0552 & -0.0520 & -0.0260 & -0.0282 & -0.1206 & -0.0901 \\
\hline & -0.4085 & -0.4304 & -0.4654 & -0.5033 & -0.4120 & -0.4120 & -0.6023 & -0.5318 \\
\hline & 0.4114 & 0.4456 & 0.3995 & 0.4097 & 0.3969 & 0.3854 & 0.4409 & 0.3946 \\
\hline \multirow[t]{3}{*}{0.75} & -0.2447 & 0.3123 & -0.2498 & 0.3718 & -0.2208 & 0.4323 & -0.2096 & 0.3782 \\
\hline & -0.8362 & -0.2455 & -0.9202 & -0.3446 & -0.8281 & -0.2272 & -0.9726 & -0.2552 \\
\hline & 0.3237 & 0.7988 & 0.3299 & 1.1334 & 0.3664 & 1.4876 & 0.3977 & 1.5732 \\
\hline \multirow[t]{3}{*}{0.95} & -0.8230 & 1.1721 & -0.8410 & 1.2731 & -0.8513 & 1.7145 & -0.8104 & 1.6691 \\
\hline & -2.3601 & -0.0422 & -3.6114 & -0.1570 & -2.5309 & 0.3175 & -2.6314 & 0.1740 \\
\hline & 0.2966 & 3.2214 & 0.4896 & 3.4577 & 0.3208 & 3.6196 & 0.7097 & 3.7097 \\
\hline
\end{tabular}

This table reports the estimated parameter values for the QSETAR model shown in Eq. (4) where $p=1$, $d=1$, and $w_{1}$ is the median of the cryptocurrency return distribution. For each value of $\tau$, where $\tau=$ $0.05,0.25,0.50,0.75,0.95$, we report the means of the posterior distributions of parameters $\beta_{11}$ and $\beta_{21}$ (first row) along with the corresponding credible intervals which are shown below the corresponding mean estimates (second and third rows). Bold font is used to highlight statistical significance 
both Bouri et al. (2019) and Cagli (2019) report the existence of price explosivity and coexplovisivity among major cryptocurrencies, their papers stop short of examining the trigger behind the explosive behaviour. Our QSETAR estimation results show that the explosive behaviour is prompted by strong conviction by the market participants. Following previous day's price increase, the prices of Bitcoin and Ripple become explosive when their returns reach $8.71 \%$ and $10.43 \%$, respectively. As for Stellar, the explosive behaviour is triggered only when the return reaches $11.66 \%$ and the previous day's return cross the median value of around $-0.35 \%$ from below.

The evidence shows that the explosive price behaviour was triggered in a number of occasions. To provide a number of examples, we can report that Bitcoin experienced a burst of explosivity on 22nd and 23rd November 2011, that is one day after the previous day's return crossed the threshold of zero (i.e., moving from negative to positive) from $-4.8 \%$ on 20 th November to $5.8 \%$ on 21 st November. During the two days between 22 nd and 23rd November 2011, the returns on Bitcoin jumped by around 34\%, having reached $15.2 \%$ on 22 nd November and then rallied a further $18.7 \%$ on 23rd November. Similarly, Ripple showed explosive behaviour from 24th November 2013, namely one day after the previous day's return crossed the $w_{1}=0$ threshold, gaining approximately $112 \%$ during the threeday period between 24th and 26th November 2013. The explosive behaviour of Stellar was triggered on 4th May 2017 when a sharp market rally occurred one day after the previous day's return crossed the median return from below, leading to four consecutive days of price advance which resulted in the holding-period return of over $200 \%$. All of the explosive episodes described share a common trait: it is triggered when the return reaches the 95 th quantile of the return distribution one day after the return crosses the threshold from below.

Table 7 shows that the delay parameter varies according to the quantile level. Our results highlight, as well, that the delay parameters are almost identical either when the threshold is $w_{1}=0$ or the median return. Moreover, when $\tau=0.95$, we find $d^{\tau}=1$ for all returns, which suggests the following: it takes one day after the cryptocurrency return crosses the threshold (i.e., 0 and the median return, depending on the specification) for the autoregressive coefficient (i.e,. the market reaction to the previous day's price information) to switch from $\beta_{11}$ to $\beta_{21}$ if the return crosses the threshold from below and from $\beta_{21}$ to $\beta_{11}$ if the return crosses the threshold from above.

To demonstrate the superior performance of the QSETAR model, we compare its coverage probabilities of the estimated quantiles with those calculated using the conventional SETAR model whose focus is on the mean return. A good model is expected to deliver the estimated coverage probabilities of the quantile estimates that are closer to the actual quantile level $\tau$. We estimate the SETAR model with the same specification as the QSETAR in Eq. (4) as follows:

$$
r_{t}=\sum_{i=1}^{2}\left(\beta_{i 0}+\beta_{i 1} r_{t-1}+h_{i} \varepsilon_{t}\right) I_{\left[r_{t-1} \in \Omega_{i}\right]}
$$

where $\varepsilon_{t} \sim N(0,1)$ and $h_{i}^{2}$ is the conditional variance of $r_{t}$ in the $i$ th regime.

We report the coverage probabilities of the estimated quantiles for both the QSETAR and the SETAR models in Table $8 .^{7}$ Our results reveal that the QSETAR model performs better than the SETAR model according to the the coverage probabilities-especially at the 25 th

\footnotetext{
7 The coverage probability is estimated as follows. Let $q_{r_{t} \mid \mathbf{r}_{t-1}}^{\tau_{i}}$ be the $\tau_{i}$ th conditional quantile of $r_{t}$, where $t=1, \ldots, n$. Let $n_{1}^{\tau_{i}}=\sum_{t=1}^{n} I\left[r_{t} \leq q_{r_{t} \mid \mathbf{r}_{t-1}}^{\tau_{i}}\right]$, where $I[$.$] is the indicator function. Then the coverage$ probability of the $\tau_{i}$ th quantile estimates is estimated by $n_{1}^{\tau_{i}} / n$.
} 
Table 7 Estimated values of the delay parameter $d$

Table 8 Coverage probabilities for the quantile estimates from QSETAR and SETAR

\begin{tabular}{lllll}
\hline$w_{1}=0$ & & & & \\
\hline$\tau$ & Bitcoin & Litecoin & Ripple & Stellar \\
\hline 0.05 & 1 & 2 & 2 & 2 \\
0.25 & 2 & 3 & 2 & 3 \\
0.50 & 3 & 3 & 3 & 3 \\
0.75 & 2 & 2 & 1 & 2 \\
0.95 & 1 & 1 & 1 & 1 \\
\hline
\end{tabular}

$w_{1}=$ median return

\begin{tabular}{lllll}
\hline$\tau$ & Bitcoin & Litecoin & Ripple & Stellar \\
\hline 0.05 & 1 & 2 & 2 & 2 \\
0.25 & 2 & 3 & 2 & 3 \\
0.50 & 3 & 3 & 3 & 3 \\
0.75 & 2 & 2 & 2 & 2 \\
0.95 & 1 & 1 & 1 & 1
\end{tabular}

This table shows the estimated values of $d$-when the threshold value is either $w_{1}=0$ (top panel) or the median return (bottom panel)-for the best estimated models according to the AIC

\begin{tabular}{lllllll}
\hline$\tau$ & 0.05 & 0.25 & 0.50 & 0.75 & 0.95 & RMSE \\
\hline QSETAR & & & & & & \\
Bitcoin & 0.0358 & 0.2181 & 0.5127 & 0.7660 & 0.9624 & 0.0423 \\
Litecoin & 0.0313 & 0.2266 & 0.5032 & 0.7807 & 0.9670 & 0.0463 \\
Ripple & 0.0304 & 0.2226 & 0.5150 & 0.7836 & 0.9660 & 0.0524 \\
Stellar & 0.0283 & 0.2261 & 0.5131 & 0.7686 & 0.9679 & 0.0434 \\
SETAR & & & & & & \\
Bitcoin & 0.0355 & 0.1197 & 0.5066 & 0.8821 & 0.9660 & 0.1869 \\
Litecoin & 0.0343 & 0.1422 & 0.5107 & 0.8732 & 0.9649 & 0.1654 \\
Ripple & 0.0322 & 0.1346 & 0.5407 & 0.8703 & 0.9566 & 0.1726 \\
Stellar & 0.0380 & 0.1626 & 0.5257 & 0.8455 & 0.9535 & 0.1326 \\
\hline
\end{tabular}

This table reports the coverage probabilities for the quantile estimates from QSETAR and SETAR, where the coverage probability is estimated as follows. Let $q_{r_{t} \mid \mathbf{r}_{t-1}}^{\tau_{i}}$ be the $\tau_{i}$ th conditional quantile of $r_{t}$, where $t=$ $1, \ldots, n$. Let $n_{1}^{\tau_{i}}=\sum_{t=1}^{n} I\left[r_{t} \leq q_{r_{t} \mid \mathbf{r}_{t-1}}^{\tau_{i}}\right]$, where $I[$.$] is the indicator$ function. Then the coverage probability of the $\tau_{i}$ th quantile estimates is estimated by $n_{1}^{\tau_{i}} / n$

and the 75th quantiles - and the root-mean-square error (RMSE). Specifically, the RMSE results also suggest that the QSETAR model outperforms the SETAR model and therefore better suits the examination of price explosivity in the cryptocurrency markets. 


\section{Concluding remarks}

In this paper, we characterise the bubble-like behaviour of prices of four major cryptocurrencies using the QSETAR model of Cai and Stander (2008). The technique is capable of characterising the behaviour of nonstationary time series with very large, but not necessarily symmetric, variations. We experiment with two threshold values: the cryptocurrencies' median returns and zero. Models for each cryptocurrency in the sample are subject to rigorous diagnostic tests. The results show that the QSETAR models fit the data very well and that they outperform the conventional SETAR model.

Our empirical analysis reveals that the explosive behaviour in the markets for Bitcoin, Ripple, and Stellar originates from the extreme upper tails of the return distributions. Specifically, our results suggest that one day following a price increase, explosivity is triggered when the market rallies are sufficiently strong, putting the cryptocurrency returns in the 95th quantile of the return distributions. We find no evidence of price explosivity in the markets for Litecoin.

The findings in this article echo concerns raised by researchers such as Gandal et al. (2018) that cryptocurrency markets appear to be prone to excessive speculation and price manipulation. The extreme price moves frequently observed in the markets seem to suggest that market participants behave irrationally, reacting strongly to unanticipated information, thereby causing unwarranted, sustained periods of strong market rallies. The existence of price explosivity is perhaps the alluring quality of cryptocurrencies which have attracted investors' attention. As rising prices become evident, more cryptocurrency traders joins the rallies, putting upward pressure on prices, leading to frothy markets.

Acknowledgements We are very grateful to the Editor and two anonymous reviewers for their helpful comments.

Open Access This article is licensed under a Creative Commons Attribution 4.0 International License, which permits use, sharing, adaptation, distribution and reproduction in any medium or format, as long as you give appropriate credit to the original author(s) and the source, provide a link to the Creative Commons licence, and indicate if changes were made. The images or other third party material in this article are included in the article's Creative Commons licence, unless indicated otherwise in a credit line to the material. If material is not included in the article's Creative Commons licence and your intended use is not permitted by statutory regulation or exceeds the permitted use, you will need to obtain permission directly from the copyright holder. To view a copy of this licence, visit http://creativecommons.org/licenses/by/4.0/.

\section{References}

Bariviera, A. F. (2017). The inefficiency of bitcoin revisited: A dynamic approach. Economics Letters, 161, $1-4$.

Baur, D. G., \& Dimpfl, T. (2018). Asymmetric volatility in cryptocurrencies. Economics Letters, 173, $148-151$.

Baur, D. G., Hong, K., \& Lee, A. D. (2018). Bitcoin: Medium of exchange or speculative assets? Journal of International Financial Markets, Institutions and Money, 54, 177-189.

Borri, N. (2019). Conditional tail-risk in cryptocurrency markets. Journal of Empirical Finance, 50, 1-19.

Bouri, E., Shahzad, S. J. H., \& Roubaud, D. (2019). Co-explosivity in the cryptocurrency market. Finance Research Letters, 29, 178-183.

Brooks, S. P. (1998). Markov chain Monte Carlo method and its application. The Statistician, 47, 69-100.

Cagli, E. C. (2019). Explosive behavior in the prices of Bitcoin and altcoins. Finance Research Letters, 29 , 398-403.

Cai, Y., \& Stander, J. (2008). Quantile self-exciting threshold autoregressive time series models. Journal of Time Series Analysis, 29, 186-202.

Chaim, P., \& Laurini, M. P. (2018). Volatility and return jumps in bitcoin. Economics Letters, 173, $158-163$. 
Corbet, S., Lucey, B., \& Yarovaya, L. (2018a). Datestamping the Bitcoin and Ethereum bubbles. Finance Research Letters, 26, 81-88.

Corbet, S., Meegan, A., Larkin, C., Lucey, B., \& Yarovaya, L. (2018b). Exploring the dynamic relationships between cryptocurrencies and other financial assets. Economics Letters, 165, 28-34.

Cretarola, A., \& Figà-Talamanca, G. (2021). Detecting bubbles in Bitcoin price dynamics via market exuberance. Annals of Operations Research, 299, 459-479.

Fry, J. (2018). Booms, busts and heavy-tails: The story of Bitcoin and cryptocurrency markets? Economics Letters, 171, 225-229.

Gandal, N., Hamrick, J. T., Moore, T., \& Oberman, T. (2018). Price manipulation in the Bitcoin ecosystem. Journal of Monetary Economics, 95, 86-96.

Gkillas, K., \& Katsiampa, P. (2018). An application of extreme value theory to cryptocurrencies. Economics Letters, 164, 109-111.

Gronwald, M. (2019). Is Bitcoin a commodity? On price jumps, demand shocks, and certainty of supply. Journal of International Money and Finance, 97, 86-92.

Gronwald, M. (2021). How explosive are cryptocurrency prices? Finance Research Letters, 38, 101603.

Hudson, R., \& Urquhart, A. (2021). Technical trading and cryptocurrencies. Annals of Operations Research, 297, 191-220.

Ji, Q., Bouri, E., Lau, C. K. M., \& Roubaud, D. (2019). Dynamic connectedness and integration in cryptocurrency markets. International Review of Financial Analysis, 63, 257-272.

Katsiampa, P. (2017). Volatility estimation for Bitcoin: A comparison of GARCH models. Economics Letters, $158,3-6$.

Katsiampa, P., Corbet, S., \& Lucey, B. (2019a). High frequency volatility co-movements in cryptocurrency markets. Journal of International Financial Markets, Institutions and Money, 62, 35-52.

Katsiampa, P., Moutsianas, K., \& Urquhart, A. (2019b). Information demand and cryptocurrency market activity. Economics Letters, 185, 1-5.

Khuntia, S., \& Pattanayak, J. K. (2018). Adaptive market hypothesis and evolving predictability of bitcoin. Economics Letters, 167, 26-28.

King, T., \& Koutmos, D. (2021). Herding and feedback trading in cryptocurrency markets. Annals of Operations Research, 300, 79-96.

Koenker, R. (2005). Quantile Regression. Cambridge University Press.

Koutmos, D. (2018). Return and volatility spillovers among cryptocurrencies. Economics Letters, 173, 122127.

Nadarajah, S., \& Chu, J. (2017). On the inefficiency of Bitcoin. Economics Letters, 150, 6-9.

Panagiotidis, T., Stengos, T., \& Vravosinos, O. (2019). The effects of markets, uncertainty and search intensity on bitcoin returns. International Review of Financial Analysis, 63, 220-242.

Scaillet, O., Treccani, A., \& Trevisan, C. (2018). High-frequency jump analysis of the bitcoin market. Journal of Financial Econometrics pp. 1-24.

Urquhart, A. (2016). The inefficiency of Bitcoin. Economics Letters, 148, 80-82.

Wei, W. C. (2018). Liquidity and market efficiency in cryptocurrencies. Economics Letters, 168, 21-24.

Yu, P. L. H., Li, W. K., \& Jin, S. (2010). On some models for value at risk. Econometric Reviews, 29, 622-641.

Publisher's Note Springer Nature remains neutral with regard to jurisdictional claims in published maps and institutional affiliations. 